Gene Identification and Analysis: An Application

of Neural Network-based Information Fusion

$$
\text { CONF- } 9608120-2
$$

Sherri Matis, Ying Xu, Manesh B. Shah, Richard J. Mural ${ }^{1},{ }_{1}$ and Edward C. Uberbacher J.R. EINSTEIN

Informatics Group Computer Science and Mathematics Division and 'Biology Division

Oak Ridge National Laboratory Oak Ridge, TN 37831-6364

\footnotetext{
"The submitted manuscript has been authorized by a contractor of the U.S. Government under contract No. DEAC05-96OR22464. Accordingly, the U.S.

Government retains a nonexclusive, royalty-free license to publish or reproduce the published form of this contribution, or allow others to do so, for U.S. Government purposes."
}

Invited and published paper submitted to the Foundations of Information/Decision Fusion: Applications to Engineering Problems, Washington, D.C., August 7-9; 1996.

*Research was supported by the Office of Health and Environmental Research, U.S. Department of Energy under contract No. DE-AC05-960R22464 with Lockheed Martin Energy Research Corporation. 


\section{DISCLAIMER}

Portions of this document may be illegible in electronic image products. Images are produced from the best available original document. 


\title{
Gene Identification and Analysis: An Application of Neural Network-based Information Fusion
}

\author{
Sherri Matis. Ying Xu, Manesh B. Shah, Richard J. Mural ${ }^{\dagger}$. \\ J. Ralph Einstein, and Edward C. Uberbacher \\ Informatics Group \\ Computer Science and Mathematics Division and ${ }^{\dagger}$ Biology Division \\ Oak Ridge National Laboratory \\ Oak Ridge, TN 37831-636t \\ matisgrail.epm.ornl.gov
}

\begin{abstract}
Ielentifying genes within large regions of uncharacterized DNA is a difficult undertaking and is currently the focus of many research efforts. We describe a gene localization and modeling system, called GRAIL. GRAIL is a multiple sensor-neural network based system. It localizes genes in anonymous DNA sequence by recognizing genc features related to protein-coding regions and the boundaries of coding regions, e.g. splice sites, and then combines the recognized features using a neural network system. Localized coding regions are then "optimally" parsed into a gene model. RNA polymerase II promoters can also be predicted. Through years of extensive testing, GRAIL consistently localizes about $90 \%$ of coding portions of test genes with a false positive rate of about $10 \%$. A number of genes for major genetic discases have been located through the use of GRAIL, and over 1000 research laboratories worldwide use GRAIL on regular bases for localization of genes on their newly sequenced DNA.
\end{abstract}

\section{Introduction}

One of the most fundamental questions that can be asked about a DNA sequence is whether or not it encodes protein. Localization of protein-coding regions in anonymous DNA sequence by pure biological means is both time-consuming and costly. A number of computational methods have been proposed and used to predict protein-coding regions and gene structures in the past few years $[\bar{\imath}, 21,24.8,9,10,19,6]$. Though the performance of these computational methods is currently imperfect, the computer-based approach may soon be the only one capable of providing analysis and annotation at a rate compatible with the worldwide DNA sequencing effort.

Although a number of strategies for computer gene prediction exist, the most fundarnental and general methods employ pattern recognition. These methods exploit the positional and composition bias in coding vs non coding DNA, and the distribution of amino acids in proteins. Though recognition of each of these biases prorides a useful indication of coding regions. it is unrcalistic to expect a single "perfect" indicator, given the incomplete state of our understanding of the underlying biological processes around genes. We previously proposed an approach to combine information from several coding-prediction algorithms, each designed to recognize a particular sequence property, using a neural network to provide more powerful coding recognition capabilities, and have implemented the algorithm as an e-mail server system. called GRAIL (Gene Recognition and Analysis Internet Link) [21, 16]. While GRAIL has evolved considerably since its inception in 1991 the basic design principles are retained $[22,25,27]$.

DNA is composed of 2 strands of 4 nucleotide bases and it is represented as a sequence of four letters, A, C, G, T. The chromosomes consist of long strands of DNA which are organized in discreet transcription units or genes. The entire human genome is composed of 23 chromosomes pairs composed of approximately 3 billion basepairs. The purpose of a gene is to code for a specific protein, and at the DNA level, the gene is made up of protein coding regions or exons interspersed with non-cocling DNA regions or introns, and is preceded by the promoter which contains the sequence signals to initiate transcription. When the gene is expressed, the semi-conserved signal sequences in the promoter bind proteins which transcribe the 


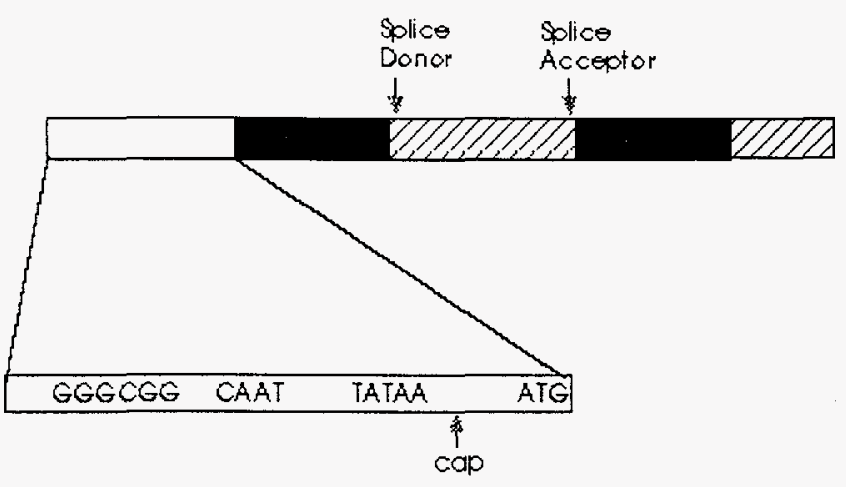

Figure 1: A schematic of a gene. The promoter region precedes the gene and is represented by an open rectangle. The sequence elements are within the promoter region. Each solid rectangle represents an exon and the diagonally striped rectangles represent introns or inter-genic regions. The boundaries of an intron are donor and acceptor junctions.

gene into a primary RNA transcript. Additional signals, such as splice donors or accepters are found at the boundaries of the introns and direct their removal during RNA processing generating a mature messenger RNA in which the protein coding region is uninterrupted. Figure 1 is a schematic of a chromosomal or genomic DNA secuence showing the chromosomal organization of genes and their features.

To determine the likelihood that a DNA segment is an exon involves measuring coding potentials of the region and evaluation of the strength of boundary signals of the region, e.g., the strength of potential splice junctions bounding the region. A number of coding measures have been proposed based on the frequency of nuclcotide "words" of a fixed length. Different types of DNA sequence (exons, introns, etc.) have different distributions of word occurrence [4]. In GRAIL, we have used a frame-dependent 6-tuple preference model [21] and a $5^{\text {th }}$ order non-homogeneous Markov chain model [2] to measure coding potentials. A number of measures including a 5 -tuple preference model, longdistance correlations between single bases, etc. have been used to measure the strength of a potential splice junction. These measures along with a number of correction factors are fed into a neural network for the final exon candidate evaluation. This neural network is trained, based on empirical data, to effectively weigh the various features in scoring the possibility of each sequence segment (candidate) being an actual exon. The use of empirical data for training allows the system to optimally utilize each feature in the presence of the others, without a priori assumptions about the independence of the features or their relative strengths.

Gene modeling involves selecting a set of most probable exon candidates that are spliceable to each other. While the neural network scores an exon candidate based on local information, the gene modeling procedure makes the final exon prediction based on more global information, i.e., whether exon candidates are spliceable or not in addition to the neural network scores.

The GRAIL gene recognition algorithm can be outlined as the following four steps. (1) Candidate generation. The algorithm first generates a large candidate pool consisting of all possible exon candidates. (2) Improbable candidate elimination. I series of heuristic rules, each of which defines some necessary conditions a probable exon candidate should satisfy, are used to eliminate majority of the improbable candidates. (3) Candidate evaluation. The candidates which have passed the rules are then evaluated by a pre-trained neural network. (4) Gene modeling. The algorithm selects, from the pool of scored exon candidates, a set of highest scoring candidates such that the adjacent candidates are spliceable to form a gene model.

Four types of exons are recognized based their different boundary signals. We use the internal exons as examples to explain the basic ideas of exon recognition. Other types of exons, initial, terminal and singleexon, can be recognized similarly. An internal exon is bounded from left by an acceptor splice junction and from right by a donor splice junction.

Promoters are recognized by combining statistical information for the sequence signals, the TATA box, GC signal, CAAT box, the cap and translation initiation sites, found within or near to promoters, with distance information for signal pairs. All of these scores are input to a back propagation neural network. The output of the neural network is then evaluated using a set of biologically relevant rules which incorporate the gene model, predicted by GRAIL, with known promoter-exon constraints.

\section{Splice Junction Recognition}

Evaluation of the donor and acceptor splice junctions is used in each of the first three steps of the GRAIL gene recognition algorithm. GRAIL recognizes acceptor junctions having the usual YAG (i.e. CAG or $T A G)$ consensus, as well as the non-standard AAG consensus, and also recognizes donor junctions containing the GT consensus. 
Recognition of donor and aceeptor splice junctions remains an imprecise art. due to a very signiticant background of non-functional sequences containing a splice consensus. Our recognition method is based on a number of relative frecuency measures of nucleoticle "words" appearing in the neighborhood of true splice sites versus false splice sites (containing minimal splice consensus) as each of those measures exhibits some discriminative power among truc and false splice junctions. A large set of true and false splice sites are used to calculate these frequencies. As a result, a profile of frequencies is obtained for true and false splice sites. respectively. Then a rector of scores can be obtained for each true or false splice site based on the calculated protiles. For each of the three types of splice junctions mentioned above, a feed-forward neural network is trained using the standard back-propagation learning algorithm. based on these vectors and their correspondling true or false labelings. to score a splice junction as being a true or false site. The neural network consists of seren inputs. one hidden later of 3 nodes and one one output.

The seren frequency measures used in the $\mathrm{YAG}$ ateceptor neural network recognition system are given as follows. Let $a_{-60} \ldots a_{35}$ represent the DNA segment containing a YAG consensus with $\iota_{0} a_{1} \iota_{2}=\mathrm{YAG}$.

$$
\sum_{i=-23}^{-1} \log \left(F_{i}^{i}\left(a_{i} \ldots a_{i+1}\right) / F_{f}^{i}\left(a_{i} \ldots a_{i+1}\right)\right)
$$

where $F_{f}^{i}()$ and $F_{f}^{i}()$ represent the positionally dependent (position i) 5-tuple frequencies in true and false splice junction regions, respectively.

(2)

$$
\sum_{i=-27}^{0} \log \left(F_{t}\left(a_{i}\right) / F_{f}\left(a_{i}\right)\right)+\sum_{i=3}^{1} \log \left(F_{t}\left(a_{i}\right) / F_{f}\left(a_{i}\right)\right) .
$$

where $F_{t} 0$ ) and $F_{f}()$ are defined similarly to (1) except that they are not positionally dependent.

(3)

$$
\sum_{i=-27}^{0} P Y\left(a_{i}\right) \sqrt{i+28}
$$

where $P Y^{-}\left(a_{i}\right)$ is 1 if $a_{i}$ is a pyrimidinc (C or $T$ ) otherwise 0.

(4) The (normalized) distance between $a_{0}$ and the nearest upstream YAG.
(5)

$$
\sum_{i=-17 i}^{1} \sum_{j \geq i}^{1} \log \left(F_{t}^{i}\left(a_{i} a_{j}\right) / F_{f}^{i}\left(a_{i} a_{j}\right)\right)
$$

where $F_{f}^{i}()$ and $F_{f}^{i}()$ are defined similarly to (1).

$(6,7)$ Coding potentials in regions of $a_{-60} \ldots a_{-1}$ and $a_{3} \ldots a_{35}$ measured using a frame-dependent 6 -ruple preference model (see next section). This is to give an indication of a transition between noncoding and coding seruences.

Acceptors with non-standard $A . A G$ consensus are recognized using basically the same measures but with clifferent frequency protiles. A separate neural network was trained based for this type of aceeptor. Similarly. donor splice junctions are recognized.

After eraluating all potential splice junctions GRAIL gencrates an exon candidate pool. Each exon candidate is a DNA segment with an open translation frame bounded by a pair of potential acceptor and donor junctions with scores larger than defined thresholds. Typically a few thousand of candidates are generated on a DNA sequence of 10000 bases long. In the second step of the GRAIL gene recognition algorithn. the splice junction scores combined with several coding potential scores are used to design a number of heuristic rules. Each of these rules defines some necessary conditions that a probable exon candidate shonld satisfy. On arerage application of these rules eliminates over $90 \%$ of the original candidates with less than $3 \%$ of true exons being removed: Hence it greatly simplifies the learning process in the neural network eralnation step, and allows the neural network learning to focus on situations where the decision is more difficult.

\section{Gene Recognition}

A coding DXt cucodes protein by encoding cach anino acid of the protein into a triplet of nucleotides. also called a codor. Recognition of a coding region essentially involves a determination of whether the DNA sequence can be partitioned into segments of three and this sequence of nucleotide triplets may possibly correspond a "valid" protein, a sequence of amino acids. A number of models have been proposed to measure the coding potential of a DNA sequence, based on the distribution of consecutive amino acids in a protein. GRAIL uses two of those models, a frame dependent 6-tuple preference model [21] and a $5^{\text {th }}$ order nonhomogencous Markov chain model [2], as basic coding measures. The coding of amino acids in nucleotide 
triplets moans that there are three possible ways to translate a DAA to protein. i.e. the three possible translation frames (two of which are incorrect).

\subsection{Feature extraction}

The frame dependent 6-tuple preference model consists of three preference values, $p f_{0}(X), p f_{1}(X)$. $p f_{2}(x)$. for cach of the 4096 possible 6 -tuples $X$, which arc detined as follows:

$$
p f_{r}(I)=\log \frac{f_{r}(X)}{f_{n}(X)} \text {, for } r=0,1,2 \text {. }
$$

where $f_{r}\left(X^{-}\right)$is the frequency of 6 -tuple $X$ appearing in a coding region and in the actual translation frame $+r$, for $r=0.1,2$, and $f_{n}(X)$ is the frequency of $X$ appearing in a noncoding region. In GRAIL, all the 6-tuple frecpuencies were calculated from a large set of DXI seruences ${ }^{1}$.

Let $a_{1} \ldots a_{n}$ be a DNA sequence of $u$ bases long. The preference model calculates the coding potential of a segnent $t_{k} \ldots t_{m}$ in each of the three possible translation frames, $r=0.1,2$, as follows.

$$
\begin{aligned}
p f_{r}\left(a_{k} \ldots a_{m}\right) & =\left(p f_{(k+5-r) \bmod 3}\left(a_{k} \ldots a_{k+5}\right)\right. \\
& +p f_{(k+6-r) \bmod 3}\left(a_{k+1} \ldots a_{k+6}\right) \\
& +p f_{(k+7-r) \bmod 3}\left(a_{k+2 \ldots a_{k+7}}\right)+\cdots \\
& +p f_{(m-r) \bmod 3} \frac{a_{m-5} \ldots a_{m}}{m-k+1}
\end{aligned}
$$

where mod is the modulo function.

Under the assumption that a DNA forms a $5^{\text {th }}$ order non-homogeneous Markov chain. GRAIL uses the Bayes formula to measure the coding potential of a DNA segment $a_{k} \ldots a_{m}$ in each of the three possible translation frames, $r=0,1,2$, as follows.

$$
\begin{aligned}
& P_{r}\left(\operatorname{coding} \mid a_{k} \ldots a_{m}\right)= \\
& \frac{P_{r}\left(a_{k} \ldots a_{m} \mid \operatorname{coding}\right)}{\sum_{f=0}^{2} P_{f}\left(a_{k} \cdots a_{m} \mid \text { coding }\right)+C P\left(a_{k} \cdots a_{m} \mid \text { noncoding }\right)},
\end{aligned}
$$

where by the Markov chain assumption,

$$
\begin{aligned}
& P_{r}\left(a_{k} \ldots a_{m} \mid \operatorname{coding}\right)= \\
& P_{(k+5-r) \bmod 3}\left(a_{k} \cdots a_{k+4} \mid \operatorname{coding}\right) \\
& \times P_{(k+5-r) \bmod 3}\left(a_{k+5} \mid a_{k} \ldots a_{k+4, \operatorname{cod}} \operatorname{cing}\right)
\end{aligned}
$$

\footnotetext{
${ }^{1}$ The set contains 450 D.NA sequences with 462608 coding bases and 2003642 noncoding bases.
}

$$
\begin{aligned}
& \times P_{(k+6-r) \bmod 3}\left(a_{k+6} \mid a_{k+1} \ldots a_{k+5}, \operatorname{cod}(i n g) \cdots\right. \\
& P_{(m-r) \bmod 3}\left(a_{m} \mid a_{m-5} \ldots a_{m-1}, \text { coding }\right) .
\end{aligned}
$$

and

$$
\begin{aligned}
& P_{n}\left(a_{k} \ldots a_{m} \mid \text { noncoding }\right)= \\
& P_{n}\left(a_{k \ldots} \ldots a_{k+4} \mid \text { noncoding }\right) \\
& P_{n}\left(a_{k+5} \mid a_{k} \ldots a_{k+4}, \text { noncoding }\right) \\
& P_{n}\left(a_{k+6} \mid a_{k+1} \ldots a_{k+5}, \text { noncoding }\right) \ldots \\
& P_{n}\left(a_{m} \mid a_{m-5} \ldots a_{m-1}, \text { noncoding }\right) .
\end{aligned}
$$

and $C$ is the estimate of the ratio of coding versus noncoding bases in DNA, $P_{r}(X \mid Y)$ and $P_{n}(X \mid Y)$ are the conditional probabilities of $X$ in coding regions (in translation frame $+r$ ) in the presence of $Y^{-}$and in noncoding regions, respectively. These conditional probabilities can be estimated using the above $p f_{r}$ and $p f_{n}$ values.

Though not being totally independent measures, each of these two models has its own coding recognition strengths and weakness according to our test results. GRAIL uses both models as the basic coding feature extraction methods, and combines them along with other measures in the neural network coding recognition system.

Coding measures by the 6-tuple preference model and the Markov chain model are also used to devise heuristic rules for improbable exon candidate elimination in the second step of GRAIL gene recognition algorithm.

\subsection{Information fusion}

In this subsection, coding measures refer to measures of coding potential using the 6-tuple preference model and the Markov chain model. The goal of the exon recognition process is not just to discriminate exons from non-exonic regions but also to score the degree of correctness of an exon candidate that overlaps actual exons. For example, we consider a candiclate which extends past one boundary of an exon, but otherwise overlaps it, to be partially correct. To achieve this scoring, we use coding measures in the flanking arcas in addition to the coding measures of a candidate region. The rationale is that strong coding indication from the neighboring areas indicates that the candidate may be just a portion of an exon. As the candidate more closely approximates an actual exon, more noncoding elements will be included in its surrounding areas and hence the surroundings will exhibit a weaker 
coding score. GRAIL uses 60 bases on cach side of an exon candidate ats the Hanking regions.

Splice junction scores are another set of measures used to help to determine the correct exon boundaries. Though false splice junction prediction may oceur, in general true splice junctions have higher scores than nearby false splice junctions. By providing to the exon recognition neural network information from coding measures of an exon candidate. scores from flanking regions and the scores of its bounding splice junctions, GRAIL can fairly accurately score the degree of overlap (or correctness) of the candidate with the actnal underlving exon.

The recognition of coding regions using the 6 -tuple (or in general $k$-tuple, for any fixed $k$ ) method is known to lave strong dependence on the $\mathrm{G}+\mathrm{C}$ ( (bases $G$ and $C$ ) composition, and is more difficult in $G+C$ poor domains. Our recent observation on the relationship of 6 -tuple coding measures and $G+C$ composition supports this belief. If we estimate the frequencies of frame-dependent coding 6-tuples and noncoding 6-tuples in the high $\mathrm{C}+\mathrm{C}$ domain, and use these frequencics to calculate coding measures for a set of coding regions and their 60-base Hanking regions in all ranges of $\mathrm{G}+\mathrm{C}$ composition, an unexpected pattern result is shown in Figure 2. The corling measures for both the coding regions and their flanks are much lower in the $\mathrm{G}+\mathrm{C}$ poor domain compared to the $\mathrm{G}+\mathrm{C}$ rich domain. A very similar behavior is observed if the 6-tuple frequencics are collected from low G+C DNA sefuences. Interestingly, though the relative separition between coding regions and their flanking regions is similar at both cuds of the $\mathrm{G}+\mathrm{C}$ composition range. many non-exonic regions in high $\mathrm{G}+\mathrm{C}$ isochore have higher coding measure than many coding regions in $\mathrm{G}+\mathrm{C}$ poor regions. This certainly highlights the necessity to include the $\mathrm{G}+\mathrm{C}$ composition as one piece of information in the neural network information fusion process. GRAIL uses the $\mathrm{G}+\mathrm{C}$ compositions of both an exon candidate region and a 2000-base region centered around the candidate as two inputs to the neural network coding recognizer.

A schematic of the neural network used in GR.AIL is shown in Figure 3. This feed-forward neural network has 13 inputs. two hiddern layers with $t$ and 3 nodes, respectively, and one ontput.

In training the neural network, our goal is to develop a network that can score the "partial correctness" of a potential exon candidate. A simple natching function.$/()$ is used to represent the correspondence of a giren candidate with the actual exon(s) during training.

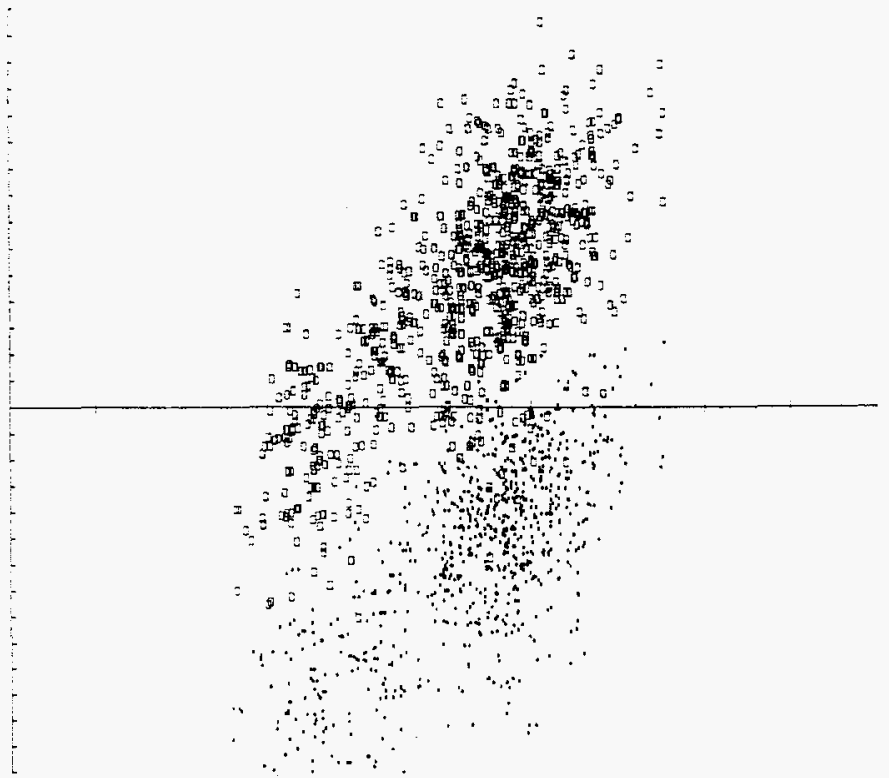

Figure 2: The $\mathrm{X}$-axis represents the $\mathrm{G}+\mathrm{C}$ composition of an exon candidate and $Y$-axis represents the 6-tuple scores measured by the frame-dependent prefcrence nodel. Each tick mark on the horizontal axis represents $10 \%$ in $\mathrm{C}+\mathrm{C}$ composition with $0 \%$ on the left and $100 \%$ on the right. The large squares represent the coding regions and the small dots represent the regions flanking coding regions.

$$
M(\text { candidate })=\frac{\sum_{i} m_{i}}{\text { length }(\text { candidate })} \frac{\sum_{i} m_{i}}{\sum_{j} \text { length }(\text { exon } j)} .
$$

where $\sum_{i} m_{i}$ is the total number of bases of the candiclate that overlap some actual exons (in the same translation frame), and $\sum_{j} \operatorname{length}\left(\mathrm{exon}_{j}\right)$ is the total length of all the exons that overlap the canclidate. Using such a function helps "teach" the neural network to discriminate candidates with different degrees of overlap with actual exons. The network was trained using the standard back-propagation algorithm on a training set containing about 2000 true, partially true and false exon candidates (a vector of features along with its corresponding $M()$ value for each candidate). All sequences used for training were from the Genome Sequence Database (GSDB) [1].

\subsection{Gene modeling}

The GRAIL gene modeling step takes as input the scored exon candidates generated by the coding recognition neural network and builds a single gene model 
Exon Candidate

Parameters

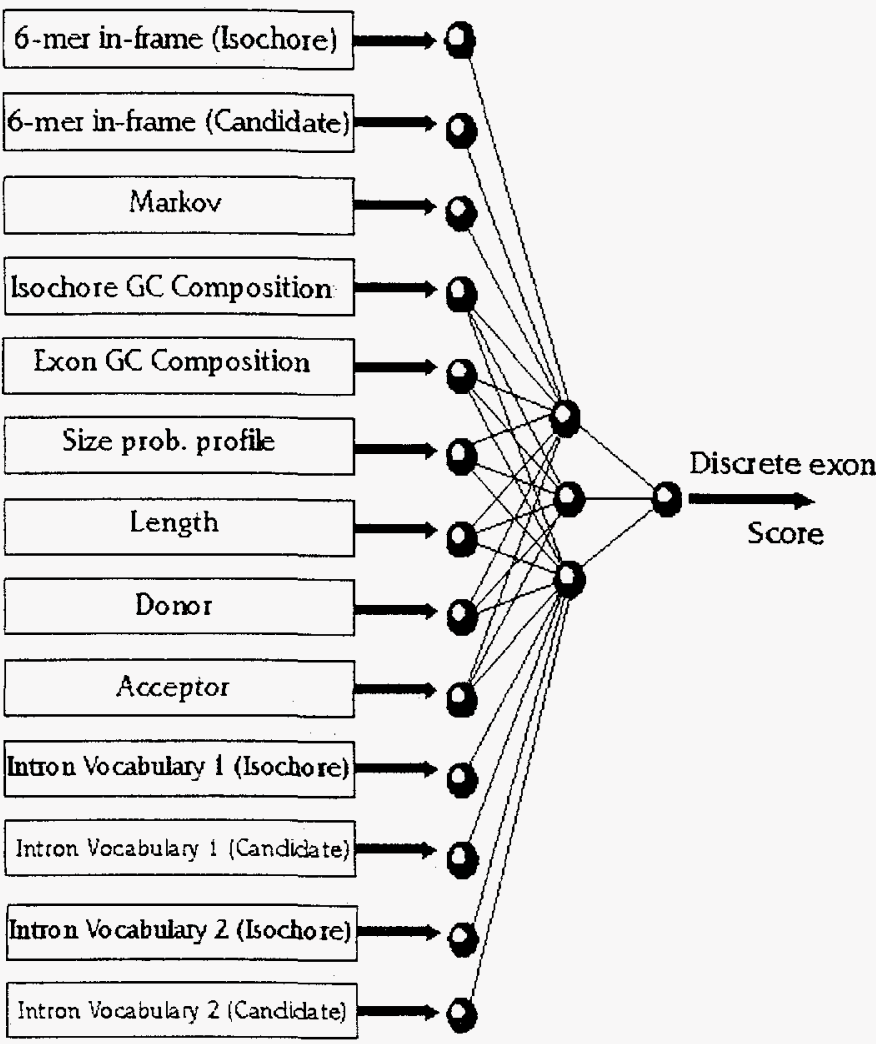

Figure 3: A schematic of the neural network for evalnating internal protein coding exons in GRAIL.

in a specified region by appending a series of nonoverlapping exon candidates under the constraints that (1) the first candiclate should start with a translation start codon ATG and the last candidate should cnd with an in-frame stop codon, TAA, TAG or TGA, (2) adjacent candiclates are spliceable (see below), (3) no in-frame stop codons can be formed when appending two adjacent exon candidates, and (4) the distance between two adjacent candidates has to be larger than the minimum intron size (60 bases are used in GRAIL).

Two candidates $a_{j} \ldots a_{k}$ and $a_{m} \ldots a_{n}, k<m$, with the preferred translation frames $r_{1}$ and $r_{2}$, respectively, are said to be spliceable if

$$
r 2=(m-k-1+r 1) \bmod 3,
$$

where the preferred translation frame refers to the frame exhibiting the highest coding potential.

GRAIL builds a gene model with the highest total neural network scores using a fast dynamic programming algorithm [24]. The basic idea of this algorithm

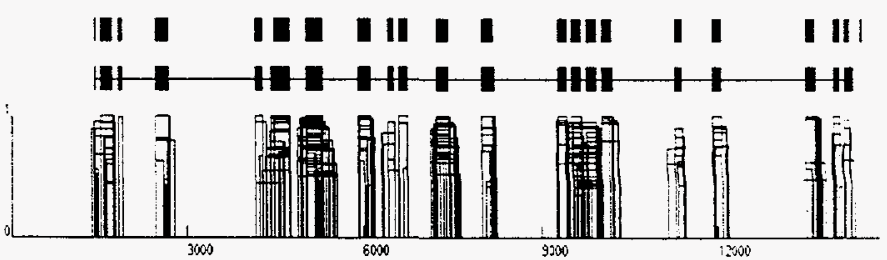

(a)

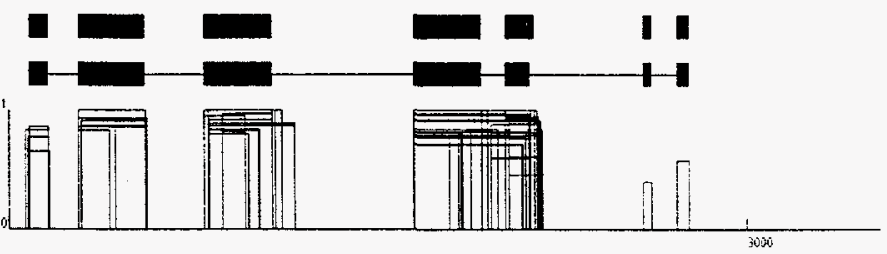

(b)

Figure 4: GRAIL gene predictions. The $\mathrm{X}$-axis is the sequence axis and the $Y$-axis is the neural net score axis. The solid bars on the top represent the aretual exons. The solid bars on the second row represent predicted exons and gene model. Each hollow rectangle denotes an exon exon candidate. (a) Sequence HUMATPGG. (b) Sequence HUMNHB27D.

is that it scans exon candidates in the increasing order of the indices of their boundaries, and builds an optimal (highest scored) partial gene model that ends with each exon candidate by extending the previous optimal partial gene models to include the current candidate. When expending an optimal partial gene model, the algorithm checks if the constraints (1) (4) are satisfied. A globally optimal solution can be obtained when the algorithm finishes scanning all the candidates.

In addition to finding a set of highest scored candidates that forms a gene model, the algorithm also helps to eliminate false exon candidates as a result of enforcing the spliceability.

Figure 4 shows two examples of GRAIL gene prediction results.

\section{Promoter Recognition}

Prediction of RNA polymerase II promoters has been attempted with only marginal success. The lack of a good method for promoter recognition reflects the variable and complex nature of the promoter region. One can simplisticly define a promoter as a collection of protein binding sites, each for a specific protein, found at a constrained distance with respect to one 
another and to the initial or starting exon. Although there are hundreds of documented protein binding sites found within different promoters. many of these sites bind protein only under certain conditions, and are found in only a small minority of promoters. The situation is further complicated by the fact that only a few binding sites are well characterized experimentally and even these can vary in sequence. Finally. because experimental analysis of promoter regions is laborious and time consuming, only in modest number of promoters have been stuclied.

In order to efficiently predict promoters. a method was developed which uses a neural network to fuse weak information with some strong constraints. Although this work is described in greater detail in [13. 14], we offer a brief description.

Promoters are detected on the basis of the presence of 5 of the most studied binding sites: the TATA box. TATAA: the GC signal. GGGCGG: the CAAT box. $C A A T$ : the cap or transcription initiation site: the translation initiation site. $A T G$ : and their distances with respect to one another[3, 5]. A candidate promoter region is defined as a region of 600 bases with an $A T A$ at position $465-475$ and is based on the format of experinentally determined promoters present in EPD $[3,13,14]$.

A vector was generated for a 13 input standard back propagation neural network. The vector was composed of seores for each of the five elements, calcurlated using a statistical frequency matrix constructed from positional nucleotide frequencies found for known functional clements and flanking regions of 10 bases. In order to use the strong distance constraint for the TATA-cap pair. The cap site was positioned so the maximum cap frequency matrix score, within 25-35 bases to the right of the TATA-box, was used for the input vector. Distance scores for the relative positions of the TAIA-GC, TATA-CAAT, TATA-cap, capATG, and GC-CAAT pairs were calculated using clistance histograms. In some cases, Multiple CAAT and or GGGCGG elements were present so the number of each of these elements was included in the input rector as well as the GC content for the 600 base region.

The output from the neural network was further refined and ralidated using a set of rules. These rules utilize gene model information from GR.AIL and known biological constraints about promoter position relative to the gene. The rules help to eliminate many false positive predictions made by the neural network. These rules are described in detail in $[13,14]$.

\section{RNA Polymerase II Promoter Network}

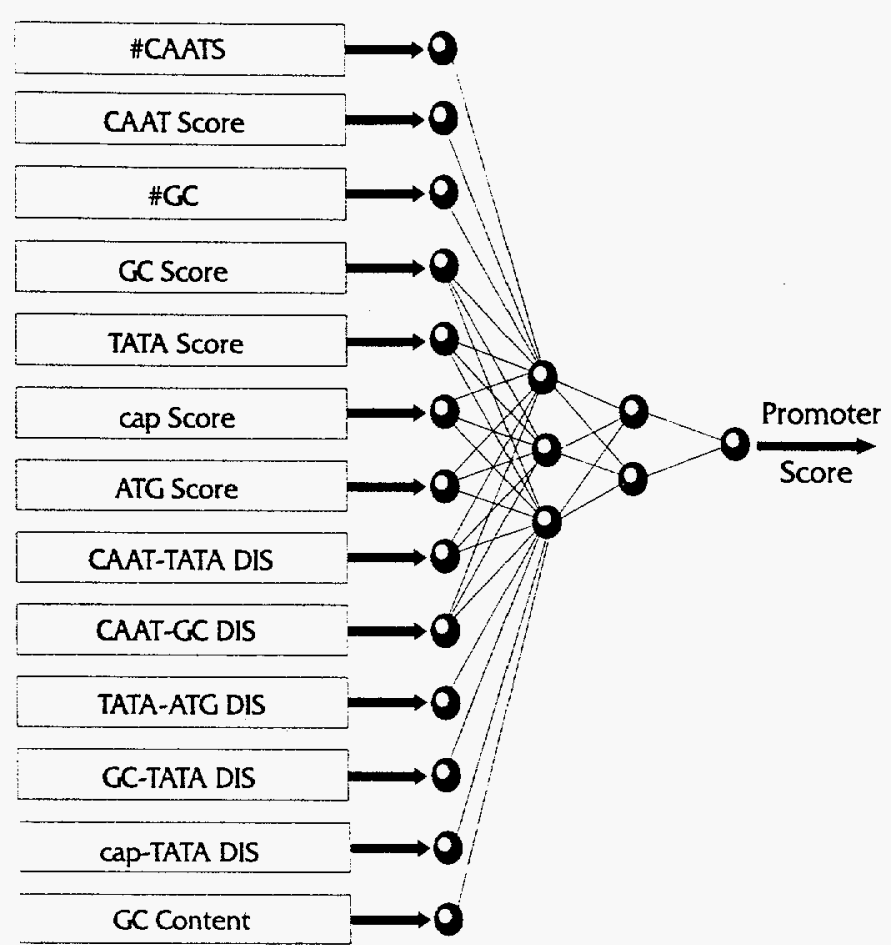

Figure 5: A schematic of the neural network used for promoter prediction. The neural network consisted of 13 input nodes 2 hidden layers of 3 and 2 hidden nodes and a single output. The network was trained and tested using a true set composed of functional promoters from EPD, the Eucaryotic Promoter Database [3] and a false set composed of 600 base regions, containing a mininal TATAA and fonnd within genes.

\section{Summary}

The performance of multi-agent systems such as GRAIL depends critically on how the information from different agents is combined. Over a dozen of exon indicators and correction factors are used in the GRAIL gene recognition process. The relationship between these quantities and the presence of exons is complicated, incomplete and clearly non-linear. To develop an effective mechanism to map these quantities, some of which may not be independent, to exon and non-exonic regions is the main goal of our research. By training neural networks with hidden layers on empirical data, GRAIL seems to have captured some of the most essential parts of this relationship based on its successful applications to gene recognition by molecular biologists worldwide over the past four years. 
By using a neural network as the basic means to combine information from different sources, we have also obtained a tlexible framework to include new information in our gene recognition system as deeper understanding and hence more information about genes are gained. Some recent work [20] has applied nenral networks to combine information from recognized gene features and data base search information in a gene recognition algorithm.

Since its service being made available to public through an email server in 1991 and also through a GUI-based client/server system in 1993, GRAIL has become one of the major tools used by molecular biologists. Over 1000 research labs worldwide use this systen to intelligently select and design biological experiments where they are most needed and most useful. Among these applications, GRAIL has helped to locate a number of genes for major genetic diseases $[11,15]$.

Through the years, GRAIL has been extensively tested on its performance of genc recognition and modcling. On a recent test on 110 Human and Mouse DNA sequences consisting of 829 exons, 134814 coding bases and 1257631 noncoding bases, GRAIL recognizes over $90 \%$ coding bases with about a $5 \%$ false positive rate as summaried in the following table. tem greatly increases the viability of the gene hunting strategies based on genomic sequencing and informatics analysis. We liave shown that the detailed structure of genes can be characterized with considerable fidelity, and expect that, in terms of providing relatively complete information about uncharacterized regions of the genome, this overall technology will fair well when compared to experimental alternatives such as exon trapping and cDNA based methods. Computational characterization of genes in their genomic sequence context will increasingly provide an important framework for understanding aspects of gene regulation and larger questions related to the functional organization of the genome.

\section{Acknowledgment}

This research was supported by the Office of Health and Environmental Research, United States Department of Energy, under contract DE-AC05-84OR21400 with Lockheed Martin Energy Systems, Inc.

Table I: GRAIL gene recognition performance.

\begin{tabular}{|c|c|c|c|c|c|c|c|c|c|}
\hline \multirow{6}{*}{$\begin{array}{l}\text { Short } \\
\text { Long } \\
\text { Total }\end{array}$} & DNA & \multicolumn{4}{|c|}{ Predictions } & \multicolumn{4}{|c|}{ Gene Modeling } \\
\hline & \# Exons & TP & $\%$ & $\mathbf{F P}$ & $\%$ & $\overline{\mathbf{T P}}$ & $\%$ & $\mathbf{F P}$ & $\%$ \\
\hline & 229 & 171 & $74.7 \%$ & 39 & $18.6 \%$ & 167 & $73.0 \%$ & 16 & $8.7 \%$ \\
\hline & 600 & 575 & $95.8 \%$ & 30 & $4.9 \%$ & 564 & $94.0 \%$ & 13 & $2.3 \%$ \\
\hline & 829 & 746 & $00.0 \%$ & 69 & $8.5 \%$ & 731 & $88.2 \%$ & 29 & $3.8 \%$ \\
\hline & \# Bases & & & & & & & & \\
\hline Total & 134814 & 122885 & $91.2 \%$ & 13048 & $9.6 \%$ & 122404 & $90.8 \%$ & 5972 & $4.7 \%$ \\
\hline
\end{tabular}

$\mathrm{TP}$ and $\mathrm{F}$ are the true and false positives, respectively. Short: 100 bases or less: Long: otherwise. In the category of Prediction, The highest scoring candidate is selected from each cluster (see Section 3.2) as the representative of the cluster.

For the largest group of RNA polymerase II promoters, $66 \%$ were detected with 1 false positive for every 23,407 bases of DNA. Although these results are not as impressive as for gene modeling, the false positive rate is much lower than other systems, which find from 1 in 1500 [12] to 1 in 6000 [17] false positives per base while finding a comparable number of correct promoters [18](58-70\% for the other systems).

The high sensitivity and specificity of the GRAIL gene recognition and modeling system and its availability through e-mail server and client/server sys- 


\section{References}

[1] H. S. Bilofsky and C. Burks, "The GenBank genetic Sequence Data bank", Nucleic Acids Res., Vol. 16, pp. 1861 - 1864, 1988.

[2] M. Borodovsky, Yu. Sprizhitskii, E. Golovanov and A. Aleksandov, "Statistical Patterns in the Primary Structures of Functional Regions in E. Coli.", Molekulyainaya Biologiya, Vol. 20, pp. 1390 - 1398, 1986.

[3] P. Bucher, "Weight Matrix Descriptions of Four Eucaryotic RNA Polymerase II Promoter Elements Derived from 502 Unrelated Promoter Sequences", J. Mol. Biol. vol. 212 pp. 563-570, 1990.

[4] J. M. Claverie, I. Sauvaget and L. Bougueleret, "ktuple Frequency Analysis: From Intron/Exon Discrimination to T-cell Epitope Mapping", Methods in Enzymology, Vol. 183, pp. 237 - 252, 1990.

[5] R. C. Conaway and J. W. Conaway, "General Initiation Factors for RNA Polymerase II",Ann. Rev. Biochem. vol. 62 pp. 161-190, 1993.

[6] S. Dong and D. B. Searls, "Genc Structure Prediction by Linguistic Methods", Genomics, Vol. 23, pp. $540-551,1994$.

[7] J. W. Fickett, "Recognition of Protein Coding Regions in DNA Sequences", Nucleic Acids Res., Vol. 10, pp. $5303-5318,1982$.

[8] M. S. Gelfand, "Computer prediction of ExonIntron Structure of Mammalian pre-mRNAs", $N u$ cleic Acids Res., Vol. 18, pp. 5865 - 5869, 1990.

[9] R. Guigo, S. Knudsen, N. Drake and T. Smith, "Prediction of Gene Structure", J. Mol. Biol, Vol. 226, pp. $141-157,1992$.

[10] G. B. Hutchinson and M. R. Hayden, "The prediction of Exons Through an Analysis of Spliceable Open Reading Frames", Nucleic Acids Res., Vol. 20, pp. $3453-3462,1992$.

[11] R. Legouis, et al., "The Candidate Gene for the X-linked Kallmann Syndrome Encodes a Protein Related to Adhesion Molecules", Cell, Vol. 67, No. 10, pp. 423 - 435, 1991.

[12] N. Mache, M. Reczho, P. Levi, and A. Hatzigeorgiou, "Multistate Time-Delay Neural Networks for the Recognition of Pol II Promoter Sequences" Proceedings of The Fourth International Conference on Intelligent Systems for Molecular Biology,
D. States, P. Agarwal, T. Gasterland, L. Hunter, and R. Smith, Eds, pp. 55, AAAI Press, 1996.

[13] S. Matis, M. Shah, R. J. Mural, and E. C. Uberbacher, "A Neural Network System for Prediction of RNA Polymerase II Promoters ", Proceedings of the First World Conference on Computational Medicine, Public Health, and Biotechnology, Vol. 1 pp. $234-252,1996$.

[14] S. Matis, Y. Xu, M. Shah, X. Guan, J. R. Einstein, R. J. Mural, and E. C. Uberbacher, "Detection of RNA Polymerase II Promoters and Polyadcnylation Sites in Human DNA Sequence", Computers and Chemistry, Vol. 20 pp. 135-140, 1995.

[15] J. Mosser, A. M. Douar, C. O. Srade, P. Kioschis, R. Feil, H. Moser, A. M. Poustka, L. J. Mandel and P. Aubourg, "Putative X-linked Adrenoleukodystrophy Gene Shares Unexpected Homology with ABC Transporters", Nature, Vol. 361, pp. 726 730 .

[16] R. J. Mural, R. J. Einstein, X. Guan, R. C. Mann and E. C. Uberbacher, "An Artificial Intelligence Approach to DNA Sequence Feature Recognition", Trends in Biotechnology, Vol. 10, pp. 66 - 69, 1992.

[17] D. Prestridge, "Predicting RNA Polymerase II Promoter Sequences using Transcription Factor Binding Sites", J. Mol. Biol. Vol 249 pp. 1995.

[18] M. Reese and F. Eeckman, "New Neural Network Algoritlims for Improved Eucaryotic Promoter Site Recognition" Genome Sci. and Tech., Vol 1 pp. P-45, 1995. Abstract

[19] E. E. Snyder and G. D. Stormo, "Identification of Coding Regions in Genomic DNA Sequences: An Application of Dynamic Programming and Neural Networks", Nucleic Acids Res., Vol. 21, pp. 607 $613,1993$.

[20] E. E. Snyder and G. D. Stormo, "Identification of Protein Coding Regions in Genomic DNA", J. Mol. Biol., Vol. 248, pp. 1 - 18, 1995.

[21] E. C. Uberbacher and R. J. Mural, "Locating Protein-coding Regions in Human DNA Sequences by a Multiple Sensors-neural Network Approach", Proc. Natl. Acad. Sci. USA, Vol. 88, pp. 11261 $11265,1991$.

[22] E. C. Uberbacher, J. R. Einstein, X. Guan and R. J. Mural, "Gene Recognition and Assembly in the 
GRAIL System: Progress and Challenges", Proceedings of The Second International Conference on Bioinformatics, Supercomputing and Complex Genome Analysis, H. A. Lim, J. W. Fickett, C. R. Cantor and R. J. Robbins, Eds, World Scientific, pp. 465 - 476, 1993.

[23] E. C. Uberbacher, Y. Xu and R. J. Mural, "Discovering and Understanding Genes in Human DNA Sequence using GRAIL", Methods in Enzymology, in press.

[24] Y. Xu, R. Mural and E. C. Uberbacher, "Constructing Gene Models from a Set of Accuratelypredicted Exons: An Application of Dynamic Programming", Computer Applications in the Biosciences, Vol. 10, pp. 613 - 623, 1994.

[25] Y. Xu J. R. Einstein, R. J. Mural, M. Shah and E. C. Uberbacher, "An Improved System for Exon Recognition and Gene Modeling in Human DNA Sequences", Proceedings of The Second International Conference on Intelligent Systems for Molecular Biology, R. Altman, D. Brutlag, P. Karp, R. Lathrop, and D. Searls, Eds, pp. 376 384, AAAI Press, 1994.

[26] Y. Xu, R. Mural and E. C. Uberbacher, "Correcting Sequencing Errors in DNA Coding Regions Using Dynamic Programming", Computer Applications in the Biosciences, Vol. 11, pp. 117 - 124, 1995.

[27] E. C. Uberbacher, Y. Xu and R. J. Mural, "Discovering and Understanding Genes in Human DNA Sequence using GRAIL", Methods in Enzymology, in press.

\section{DISCLAIMER}

This report was prepared as an account of work sponsored by an agency of the United States Government. Neither the United States Government nor any agency thereof, nor any of their employees, makes any warranty, express or implied, or assumes any legal liability or responsibility for the accuracy, completeness, or usefulness of any information, apparatus, product, or process disclosed, or represents that its use would not infringe privately owned rights. Reference herein to any specific commercial product, process, or service by trade name, trademark, manufacturer, or otherwise does not necessarily constitute or imply its endorsement, recommendation, or favoring by the United States Government or any agency thereof. The views and opinions of authors expressed herein do not necessarily state or reflect those of the United States Government or any agency thereof. 